\title{
Velocidade periférica do disco em mecanismos dosadores de sementes de milho e soja
}

\author{
Peripheral speed of the plate in seed meters of corn and soybean
}

\author{
Vilnei de Oliveira Dias ${ }^{\mathrm{I}}$ Airton dos Santos Alonço ${ }^{\mathrm{II}}$ Dauto Pivetta Carpes ${ }^{\mathrm{II}}$ \\ André Augusto Veit ${ }^{\mathrm{II}}$ Lucas Barros de Souza ${ }^{\mathrm{II}}$
}

\section{RESUMO}

\begin{abstract}
O objetivo deste trabalho foi avaliar o efeito da velocidade periférica do disco dosador de sementes em mecanismos dosadores pneumáticos e de disco alveolado horizontal, com as culturas do milho e soja, em ensaio de laboratório. Os tratamentos foram compostos por quatro velocidades periféricas $(0,09$, $0,18,0,28$ e $0,38 \mathrm{~m} \mathrm{~s}^{-1}$ ) em combinação com quatro mecanismos dosadores (dois pneumáticos de pressão negativa e dois de disco alveolado horizontal) em delineamento blocos ao acaso com três repetições. Os mecanismos foram comparados por teste de médias e, para as velocidades periféricas, traçaram-se equações de regressão. Foram avaliados os percentuais de espaçamentos aceitáveis, múltiplos e falhos entre sementes por meio do espaçamento teórico nominal resultante de cada tratamento. $O$ percentual de espaçamentos aceitáveis reduziu de maneira linear com o aumento da velocidade periférica do disco para ambas as culturas. Para a cultura do milho, os mecanismos dosadores pneumáticos apresentaram resultados superiores aos de disco alveolado nas velocidades periféricas mais elevadas. Para a cultura da soja, os dosadores pneumáticos apresentaram melhor desempenho na média dos tratamentos. De maneira geral, quanto menor a velocidade periférica do disco, melhor a regularidade de distribuição de sementes de milho e soja.
\end{abstract}

Palavras-chave: espaçamentos aceitáveis, regularidade de distribuição, semeadora de precisão, semeadora pneumática.

\section{ABSTRACT}

The purpose of this study was to evaluate the effect of peripheral speed of the disc in pneumatic and horizontal plate metering mechanisms, with corn and soybean seeds, in laboratory test. The treatments consisted of four peripheral speeds $(0.09,0.18$, 0.28 and $\left.0.38 \mathrm{~m} \mathrm{~s}^{-1}\right)$ in combination with four metering mechanisms (two pneumatic and two horizontal plate) in split-plot design with three replications. The mechanisms were compared by mean test and, for peripheral speeds, draw up regression equations. The index of the acceptable, multiple and fail between seeds through the theoretical nominal spacing's resulting from each treatment. The acceptable spacing's index decreased linearly with increasing peripheral speed of the disc to both cultures. For corn, the pneumatic metering mechanisms showed better results to the horizontal plate in higher peripheral speeds. For soybean, the pneumatic feeders performed better on the average of treatments. In general, how much lower the peripheral speed of the plate, better the regularity of seeds distribution of corn and soybean.

Key words: acceptable index, quality offeed index, precision seeder, pneumatic seeder.

\section{INTRODUÇÃO}

As máquinas para semear desempenham fundamental importância nos cultivos agrícolas anuais. Para que grandes extensões de área cultivada sejam semeadas, é fundamental o emprego de máquinas, as quais são responsáveis por acondicionar a semente de maneira adequada no solo, propiciando um bom ambiente para a germinação (KARAYEL \& OZMERZI, 2007). Velocidades de semeadura elevadas são cada vez mais utilizadas, visando a aumentar a capacidade operacional dos conjuntos mecanizados, fato que pode comprometer a qualidade do processo, comprometendo o estabelecimento e por consequência a produtividade das culturas (IVANČAN et al., 2004). Visando a um adequado estande de plantas, é necessário que as sementes

\footnotetext{
'Laboratório de Mecanização Agrícola do Pampa (LAMAP), Universidade Federal do Pampa (UNIPAMPA), Campus Alegrete, Avenida Tiarajú, 810, 97546-550, Alegrete, RS, Brasil. E-mail: vilneidias@unipampa.edu.br. Autor para correspondência.

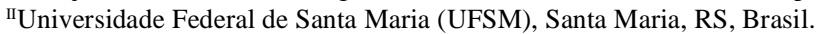


sejam dosadas de maneira correta, fato este que leva à utilização de mecanismos dosadores de precisão, que depositam estas em densidade de semeadura pré-estabelecida de acordo com a recomendação de cada cultura. Entre os diferentes tipos de mecanismos dosadores, destacam-se os de disco alveolado horizontal e os pneumáticos.

A comparação entre mecanismos dosadores de disco alveolado e pneumáticos foi estudada para a cultura da soja por JASPER et al. (2011). Os autores verificaram que o mecanismo dosador pneumático apresentou melhores resultados em comparação com o dosador de disco alveolado. Entretanto, este último não sofreu com a elevação da velocidade de deslocamento. Também para a cultura da soja, PINHEIRO NETO et al.(2008) encontraram resultados superiores para mecanismos dosadores pneumáticos quando elevada a velocidade de semeadura. A maioria dos estudos com semeadoras equipadas com estes tipos de mecanismos concentrase na velocidade de deslocamento e não na velocidade periférica do disco, como nos trabalhos de LIU et al. (2004), CELIK et al. (2007), DIAS et al. (2009) e JASPER et al. (2011). Assim, o objetivo deste trabalho foi estudar a regularidade de distribuição de sementes de milho e soja, por mecanismos dosadores pneumáticos e de disco alveolado horizontal, em quatro velocidades periféricas do disco.

\section{MATERIAL E MÉTODOS}

O trabalho foi realizado em laboratório, sobre esteira carpetada, de acordo com a norma ISO 7256/1 (ISO, 1984). Foram utilizadas sementes de milho híbrido Dekalb ${ }^{1}$ 240, peneira C2 e cultivar de soja Syngenta NK 7059 RR peneira 6,5. As características dimensionais comprimento, diâmetro e espessura foram medidas através de paquímetro digital com precisão de $0,05 \mathrm{~mm}$ em amostras de 50 sementes (YAZGI; DEGIRMENCIOGLU,
2007). Além disso, foram determinados peso de mil sementes e ângulo de repouso, além daesfericidade pela expressão mostrada por KARAYEL et al. (2004):

$$
\emptyset=\frac{(L \cdot W \cdot T)^{1 / 3}}{L} \cdot 100 \text {, onde: }
$$

$\emptyset$ é a esfericidade (\%), L, W e T referem-se a comprimento, largura e espessura da semente (mm), respectivamente. As características dimensionais são apresentadas na tabela 1 .

As sementes de milho e soja foram depositadas sobre esteira carpetada montada em estrutura metálica tubular com $17,5 \mathrm{~m}$ de comprimento e $0,15 \mathrm{~m}$ de largura, a qual é forçada a tomar a forma de "V" (formato de sulco), por dois rolos verticais posicionados junto à saída do tubo condutor de sementes. O princípio de funcionamento da esteira consiste na inversão do que ocorre no processo de semeadura em campo, onde a máquina movimentase sobre o solo; neste caso, a esteira movimenta-se sob o mecanismo dosador. A potência necessária para movimentação da esteira carpetada e acionamento dos mecanismos dosadores foi fornecida por dois motores elétricos de 2,30 e $0,36 \mathrm{~kW}$ de potência, trifásico e monofásico, respectivamente. Para promover a correta adequação à faixa necessária de rotações das fontes de potência, utilizaram-se inversores de frequência com faixas de trabalho de 1 a 1800rpm. A pressão negativa necessária à captação de sementes pelos mecanismos pneumáticos foi fornecida por bancada composta porventilador centrífugo à rotação de 3600rpm, manômetro e controlador de vácuo.

Os tratamentos foram compostos pelo fatorial dos quatro mecanismos dosadores de sementes e quatro velocidades periféricas (VP's) em delineamento blocos ao acaso, com três repetições. As VP's foram de 0,09, 0,18, 0,28 e 0,38 $\mathrm{m} \mathrm{s}^{-1}$. Foram utilizados mecanismos dosadores pneumáticosdas marcas comerciais John Deere ${ }^{1}$ e Jumil (DP1 e DP2) e de disco alveolado horizontal Stara e Semeato

Tabela 1 - Características dimensionais das sementes utilizadas nos ensaios.

\begin{tabular}{|c|c|c|c|c|}
\hline \multirow{2}{*}{ Variável } & \multirow[b]{2}{*}{ Média } & \multirow[b]{2}{*}{ Desvio padrão } & \multirow[b]{2}{*}{ Média } & \multirow[b]{2}{*}{ Desvio padrão } \\
\hline & & & & \\
\hline Comprimento (mm) & 7,4 & 0,8 & 4,2 & 0,4 \\
\hline Diâmetro (mm) & 5,1 & 0,3 & 3,9 & 0,3 \\
\hline Espessura (mm) & 2,1 & 0,3 & 3,3 & 0,5 \\
\hline Massa de 1000 sementes (g) & 280,1 & 4,1 & 110,0 & 3,5 \\
\hline Ângulo de repouso $\left(^{\circ}\right)$ & 29,8 & 3,8 & 33,4 & 1,3 \\
\hline Esfericidade (\%) & 58,2 & 5,6 & 90,0 & 6,7 \\
\hline
\end{tabular}


(DDH1 e DDH2). Os orifícios dos dosadores foram dimensionados de acordo com o ajuste do espaço livre entre orifícios e o tamanho da semente (SANTOS et al., 2003).

Auniformidade de distribuiçãolongitudinal de sementes foi avaliada de acordo com a ISO 7256/1 (ISO, 1984) pelo percentual de espaçamentos falhos ( $\geq 1,5$ vezes o espaçamento nominal), múltiplos $(\leq 0,5$ vezes o espaçamento nominal) e os demais foram classificados como aceitáveis (ISO, 1984). Os dados foram submetidos ao teste de KolmogorovSmirnov, com $\mathrm{P}<0,05$, no intuito de testar a aderência dos resíduos à distribuição normal e ao teste de Bartlett $(\mathrm{P}<0,01)$ para identificar a condição de homogeneidade das variâncias. Se não atendido o pressuposto de normalidade, os dados foram transformados de acordo com a expressão $x f=\operatorname{arcsen} \sqrt{ }(x / 100)$, em que $x f$ representa o dado transformado e $\mathrm{x}$ o dado original. Identificadas as condições de normalidade e homogeneidade, procedeu-se à análise da variância (ANOVA, com $\mathrm{P}<0,05)$. As médias foram comparadas pelo teste de Tukey com 5\% de probabilidade de erro.

\section{RESULTADOS E DISCUSSÃO}

Para o milho, apenas o percentual de espaçamentos falhos necessitou de transformação para atender a condição de normalidade (Tabela 2). Este mesmo índice não pode ser submetido ao teste de homogeneidade, por apresentar dados amostrais iguais a zero, o que impossibilita a estimativa da variância da amostra (SILVA; AZEVEDO, 2009). Para a cultura da soja, a análise de normalidade mostrou que as variáveis de distribuição aderiram à distribuição normal, assim como as variâncias se mostraram homogêneas, exceto para o percentual de espaçamentos múltiplos, que foram consideradas homogêneas pelo teste de Bartlett (Tabela 2).

Quanto ao percentual de espaçamentos aceitáveis, múltiplos e falhos entre sementes de milho, houve diferenças significativas entre os mecanismos dosadores de sementes e interação entre os tratamentos (Tabela 3). Para a VP de 0,09 $\mathrm{m} \mathrm{s}^{-1}$, não houve diferença entre os quatro mecanismos dosadores de sementes, o que também aconteceu com $0,28 \mathrm{~m} \mathrm{~s}^{-1}$ de velocidade periférica do disco. Observando as médias da interação entre os fatores mecanismos dosadores e VP's do disco, nota-se que a variável percentual de espaçamentos aceitáveis reduziu em apenas 9\% para o dosador DP1 e em 35 e $31 \%$ para os dosadores DDH1 e DDH2, respectivamente, o que ilustra uma maior capacidade de suportar maiores velocidades periféricas dos dosadores pneumáticos. Essa informação corrobora a de DIAS et al. (2009), que verificaram redução no percentual de espaçamentos aceitáveis para soja com aumento da VP de discos dosadores alveolados horizontais. A diferença entre os mecanismos dosadores se mostrou mais acentuada na VP de $0,38 \mathrm{~m} \mathrm{~s}^{-1}$, sendo que os percentuais de espaçamentos aceitáveis entre sementes de milho foram elevados, denotando boa regularidade de distribuição, o que é evidenciado na coluna das médias dos mecanismos dosadores, independente da velocidade periférica do disco dosador. $\mathrm{O}$ percentual de espaçamentos falhos foi menor para o DP1, exceto para a menor VP do disco.

As regressões polinomiais significativas foram lineares para percentual de espaçamentos aceitáveis, múltiplos e falhos (Figura 1), corroborando o trabalho de YAZGI \& DEGIRMENCIOGLU (2007), que verificaram redução na regularidade de

Tabela 2 - Resumo da análise de normalidade dos dados pelo teste de Kolmogorov-Smirnov e homogeneidade das variâncias pelo teste de Bartlett para as variáveis estudadas.

\begin{tabular}{|c|c|c|c|c|c|c|}
\hline \multirow{2}{*}{ Espaçamentos } & & \multicolumn{2}{|c|}{ Vormalidade (5\%)------------------------ } & \multicolumn{3}{|c|}{----Homogeneidade (1\%)--------------- } \\
\hline & Valor & Valor crítico & Normal & $\chi^{2}$ & $\chi^{2}$ crítico & Variâncias \\
\hline Aceitáveis & 0,08 & 0,13 & Sim & 53,58 & 30,58 & Heterogêneas \\
\hline Múltiplos & 0,09 & 0,13 & Sim & 28,22 & 30,57 & Homogêneas \\
\hline Falhos & 0,16 & 0,13 & Não & & 2 & \\
\hline Falhos $^{1}$ & 0,12 & 0,13 & Sim & & & \\
\hline Aceitáveis & 0,11 & 0,13 & Sim & 17,07 & 30,58 & Homogêneas \\
\hline Múltiplos & 0,08 & 0,13 & Sim & 32,53 & 30,58 & Heterogêneas ${ }^{1}$ \\
\hline Falhos & 0,08 & 0,13 & Sim & 21,81 & 30,58 & Homogêneas \\
\hline
\end{tabular}

Ciência Rural, v.44, n.11, nov, 2014. 
Tabela 3 -Médias dos fatores mecanismos dosadores e velocidades periféricas para as médias de espaçamentos entre sementes aceitáveis, múltiplos e falhos para a cultura do milho.

\begin{tabular}{|c|c|c|c|c|c|}
\hline \multirow{2}{*}{ Dosador } & \multicolumn{4}{|c|}{--Velocidade periférica $\left(\mathrm{m} \mathrm{s}^{-1}\right)-$} & \multirow{2}{*}{ Média } \\
\hline & 0,09 & 0,18 & 0,28 & 0,38 & \\
\hline \multicolumn{6}{|c|}{ Espaçamentos aceitáveis (\%) } \\
\hline DP1 & $84,3 \mathrm{a}$ & $83,5 \mathrm{a}$ & $78,1 \mathrm{a}$ & $76,8 \mathrm{a}$ & $80,7 \mathrm{a}$ \\
\hline DP2 & $97,9 \mathrm{a}$ & $79,9 \mathrm{ab}$ & $81,1 \mathrm{a}$ & $67,1 \mathrm{ab}$ & $81,5 \mathrm{a}$ \\
\hline DDH1 & $95,2 \mathrm{a}$ & $66,2 b$ & $78,3 \mathrm{a}$ & $61,8 \mathrm{~b}$ & $75,4 \mathrm{~b}$ \\
\hline DDH2 & $93,2 \mathrm{a}$ & $89,2 b$ & $89,2 \mathrm{a}$ & $64,3 \mathrm{ab}$ & $79,3 \mathrm{ab}$ \\
\hline \multicolumn{6}{|c|}{ Espaçamentos múltiplos (\%) } \\
\hline DP1 & $15,7 \mathrm{a}$ & $10,4 a b$ & $21,2 \mathrm{a}$ & $21,6 \mathrm{a}$ & $17,2 \mathrm{a}$ \\
\hline DP2 & $1,8 \mathrm{~b}$ & $9,8 \mathrm{ab}$ & $12,2 \mathrm{~b}$ & $16,1 \mathrm{a}$ & $10,0 \mathrm{c}$ \\
\hline DDH1 & $3,9 b$ & $15,7 \mathrm{a}$ & $10,4 \mathrm{~b}$ & $20,2 \mathrm{a}$ & $12,6 \mathrm{~b}$ \\
\hline DDH2 & $6,6 \mathrm{~b}$ & $5,8 \mathrm{~b}$ & $16,6 a b$ & $23,6 \mathrm{a}$ & $13,2 \mathrm{~b}$ \\
\hline \multicolumn{6}{|c|}{ Espaçamentos falhos (\%) } \\
\hline DP1 & $0,0 \mathrm{a}$ & $14,4 \mathrm{~b}$ & $4,8 \mathrm{~b}$ & $7,0 \mathrm{~b}$ & $6,6 \mathrm{c}$ \\
\hline DP2 & $2,8 \mathrm{a}$ & $17,7 \mathrm{~b}$ & $14,9 \mathrm{a}$ & $24,2 \mathrm{a}$ & $14,9 \mathrm{ab}$ \\
\hline DDH1 & $5,1 \mathrm{a}$ & $25,0 \mathrm{a}$ & $19,5 \mathrm{a}$ & $25,1 \mathrm{a}$ & $18,7 \mathrm{a}$ \\
\hline \multirow[t]{2}{*}{$\mathrm{DDH} 2$} & $1,2 \mathrm{a}$ & $12,7 \mathrm{~b}$ & $20,9 a$ & $20,0 \mathrm{a}$ & $13,8 \mathrm{~b}$ \\
\hline & \multicolumn{3}{|c|}{ DMS das interações } & \multicolumn{2}{|l|}{$\mathrm{CV}(\%)$} \\
\hline Aceitáveis & \multicolumn{3}{|c|}{14,2} & \multicolumn{2}{|l|}{3,9} \\
\hline Múltiplos & \multicolumn{3}{|c|}{8,9} & 11,9 & \\
\hline Falhos & \multicolumn{3}{|c|}{6,7} & \multicolumn{2}{|l|}{23,9} \\
\hline
\end{tabular}

DP1: dosador pneumático 1; DP2: dosador pneumático 2; DDH1: dosador de disco alveolado 1; DDH2: dosador de disco alveolado 2; DMS: diferença mínima significativa; médias seguidas de mesma letra minúscula na coluna não diferem pelo teste de Tukey a 5\% de probabilidade de erro; ns: não significativo. Não há DMS entre os tratamentos velocidades, por serem analisadas quantitativamente.

distribuição de sementes com aumento da velocidade periférica do disco dosador, embora os autores tenham trabalhado com menores velocidades periféricas do disco. O percentual de espaçamentos aceitáveis caiu de $92,8 \%$ para $67,5 \%$ em média para os quatro mecanismos dosadores. De forma inversa, o percentual de múltiplos cresceu de 7 para 20\%, com coeficiente de determinação igual de $99 \%$. Os resultados estão de acordo com os obtidos por BOZDOĞAN (2008), que trabalhou com três semeadoras pneumáticas e quatro velocidades periféricas do disco buscando um ponto ótimo de desempenho. Os autores comentam que quanto menor a velocidade periférica do disco, melhor o desempenho.

Para a cultura da soja, a análise da variância mostrou diferenças entre os mecanismos dosadores de sementes e interação deste fator com as quatro diferentes velocidades periféricas do disco dosador (Tabela 4). As médias do percentual de espaçamentos aceitáveis variaram de 40,5 até $88,4 \%$, relativas aos mecanismos dosadores DDH2 na velocidade periférica de $0,38 \mathrm{~m} \mathrm{~s}^{-1}$ e ao DP1 na velocidade periférica de $0,09 \mathrm{~m} \mathrm{~s}^{-1}$, respectivamente. O mecanismo dosador pneumático 1 (DP1) apresentou, em média, os melhores resultados para percentual de espaçamentos aceitáveis, enquanto que as médias dos mecanismos de disco alveolado não apresentaram diferença significativa. As médias do fator mecanismo dosador para o percentual de espaçamentos falhos não apresentaram diferenças significativas. Analisando a interação entre os mecanismos dosadores e as velocidades periféricas, pode-se notar que o mecanismo DP1 apresentou queda na qualidade de distribuição com elevação da VP de 0,09 para 0,38 $\mathrm{m} \mathrm{s}^{-1}$ de apenas $20 \%$ no percentual de aceitáveis, enquanto que o mecanismo DDH2 reduziu em $40 \%$. Este resultado corrobora o de JASPER et al. (2011), que concluíram que, mesmo com redução significativa nos espaçamentos aceitáveis de sementes, ao se elevar a velocidade de semeadura, o sistema pneumático apresentou melhores resultados em comparação ao sistema de disco alveolado horizontal.

A equação de regressão linear foi a que melhor se adaptou para o percentual de espaçamentos aceitáveis, com ajuste de $85 \%$ (Figura 2). A velocidade periférica de $0,09 \mathrm{~m} \mathrm{~s}^{-1}$ destacou-se das demais, com tendência a aumento na qualidade de distribuição com 


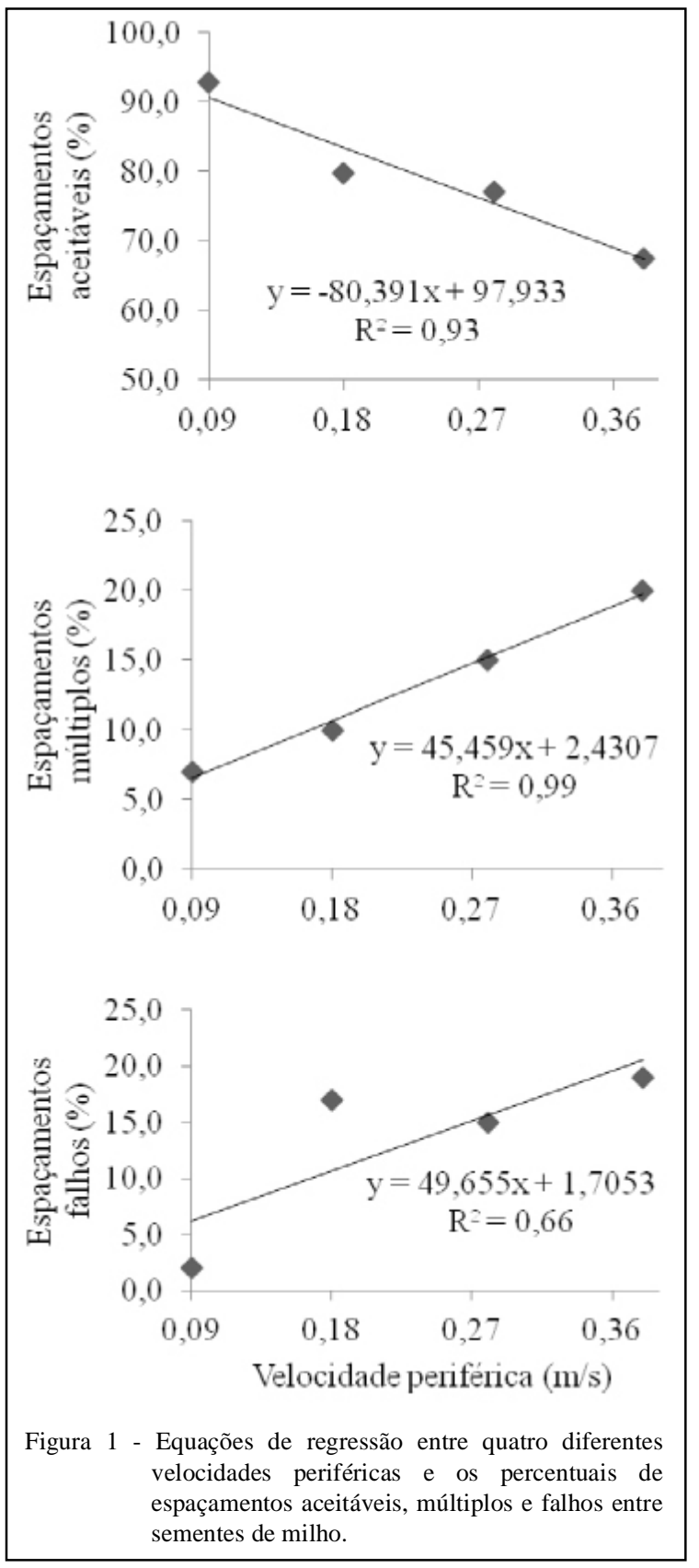

redução da velocidade. Este fato também é denotado pelos percentuais de espaçamentos múltiplos e falhos que tenderam a aumentar nas velocidades periféricas maiores que $0,18 \mathrm{~m} \mathrm{~s}^{-1}$. Tais resultados ratificam os encontrados por JASPER et al. (2011), que verificaram efeito significativo da elevação da velocidade de semeadura para até $3,33 \mathrm{~m} \mathrm{~s}^{-1}$ na variável percentual de espaçamentos aceitáveis. Por outro lado, contrastam com as conclusões de REIS et al. (2007), que não obtiveram resposta linear da regularidade de distribuição de sementes com aumento da velocidade.

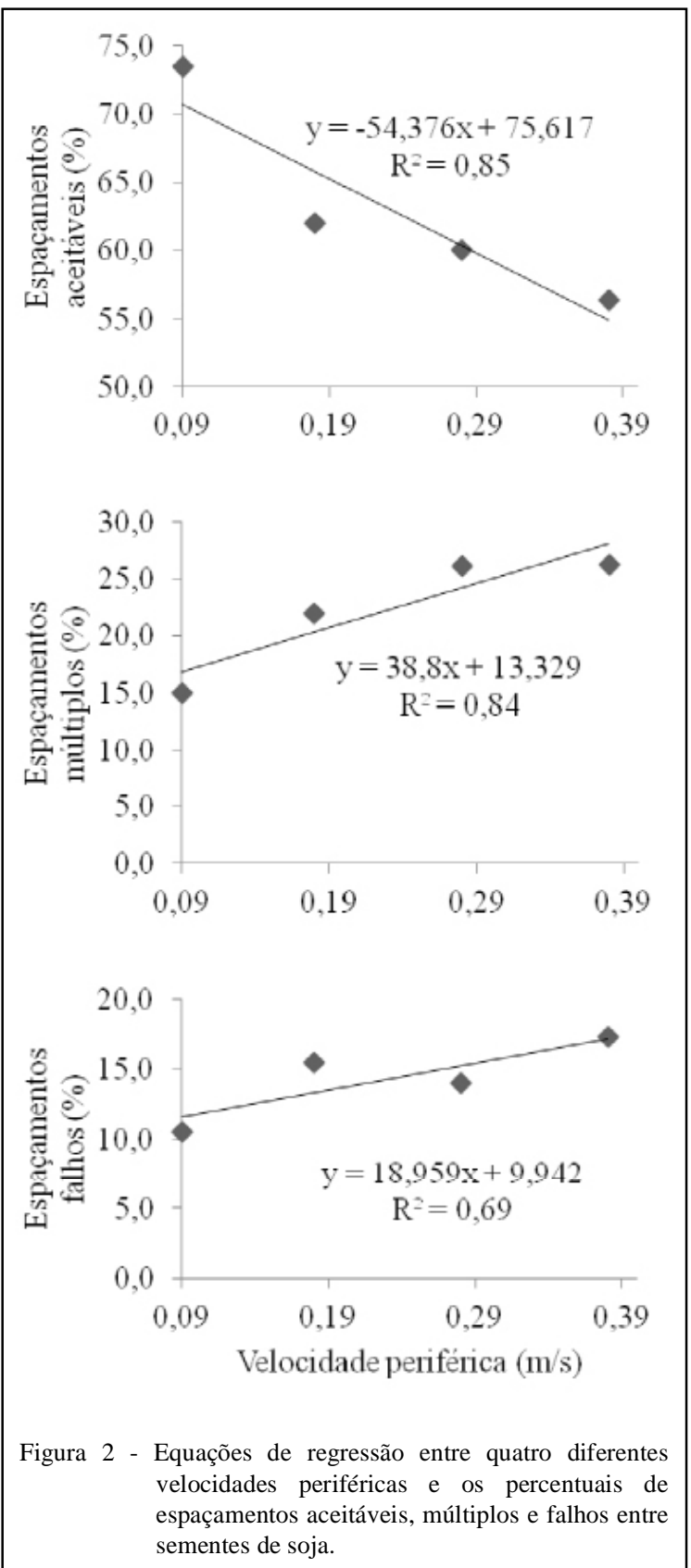

Os resultados também diferem dos reportados por KLEIN et al. (2002), que não encontraram efeito no percentual de espaçamentos duplos e falhos na semeadura direta da soja, com velocidades variando de 1,0 a $3,0 \mathrm{~m} \mathrm{~s}^{-1}$.

\section{CONCLUSÃO}

Os mecanismos dosadores de sementes avaliados apresentam diferenças na regularidade de distribuição quando elevada a velocidade periférica 
Tabela 4 -Médiasdos fatores mecanismos dosadores e velocidades periféricas para as médias de espaçamentos entre sementes aceitáveis, múltiplos e falhos para a cultura da soja.

\begin{tabular}{|c|c|c|c|c|c|}
\hline \multirow{2}{*}{ Dosador } & & eloc & $\left(\mathrm{m} \mathrm{s}^{-1}\right)$ & & \multirow{2}{*}{ Médi } \\
\hline & 0,09 & 0,18 & 0,28 & 0,38 & \\
\hline \multicolumn{6}{|c|}{ Espaçamentos aceitáveis (\%) } \\
\hline DP1 & $88,4 \mathrm{a}$ & $59,7 \mathrm{~b}$ & $72,9 \mathrm{a}$ & $69,7 \mathrm{a}$ & $72,7 \mathrm{a}$ \\
\hline DP2 & $76,6 b$ & $68,4 a$ & $69,0 \mathrm{ab}$ & $57,6 b$ & $67,9 b$ \\
\hline DDH1 & $61,8 \mathrm{c}$ & $45,2 \mathrm{c}$ & $62,4 b c$ & $57,7 \mathrm{~b}$ & $56,8 \mathrm{c}$ \\
\hline DDH2 & $67,5 \mathrm{c}$ & $56,6 \mathrm{~b}$ & $58,9 \mathrm{c}$ & $40,5 \mathrm{c}$ & $55,9 \mathrm{c}$ \\
\hline \multicolumn{6}{|c|}{ Espaçamentos múltiplos (\%) } \\
\hline DP1 & $7,3 b$ & $24,9 b$ & $7,5 \mathrm{c}$ & $13,0 \mathrm{c}$ & $13,2 \mathrm{c}$ \\
\hline DP2 & $9,5 b$ & $19,3 b$ & $14,9 b$ & $25,0 b$ & $17,1 b$ \\
\hline DDH1 & $24,9 \mathrm{a}$ & $34,5 \mathrm{a}$ & $18,7 \mathrm{~b}$ & $26,8 \mathrm{ab}$ & $26,2 \mathrm{a}$ \\
\hline $\mathrm{DDH} 2$ & $21,7 \mathrm{a}$ & $23,3 b$ & $28,0 \mathrm{a}$ & $32,4 \mathrm{a}$ & $26,3 \mathrm{a}$ \\
\hline \multicolumn{6}{|c|}{ Espaçamentos falhos (\%) } \\
\hline DP1 & $4,4 \mathrm{~b}$ & $15,4 \mathrm{ab}$ & $19,6 \mathrm{a}$ & $17,3 b$ & $14,2^{\text {ns }}$ \\
\hline DP2 & $14,0 \mathrm{a}$ & $12,4 b$ & $16,1 \mathrm{a}$ & $17,4 \mathrm{~b}$ & 15,0 \\
\hline DDH1 & $13,3 \mathrm{a}$ & $20,5 \mathrm{a}$ & $18,9 \mathrm{a}$ & $15,5 b$ & 17,0 \\
\hline \multirow[t]{2}{*}{$\mathrm{DDH} 2$} & $10,8 \mathrm{ab}$ & $20,0 \mathrm{ab}$ & $13,1 \mathrm{a}$ & $27,2 \mathrm{a}$ & 17,8 \\
\hline & \multicolumn{3}{|c|}{ DMS das interações } & $\mathrm{CV}(\%)$ & \\
\hline Aceitáveis & \multicolumn{3}{|c|}{7,5} & 4,8 & \\
\hline Múltiplos & \multicolumn{3}{|c|}{6,9} & 9,9 & \\
\hline Falhos & \multicolumn{3}{|c|}{7,9} & 22,9 & \\
\hline
\end{tabular}

DP1: dosador pneumático 1; DP2: dosador pneumático 2; DDH1: dosador de disco alveolado 1; DDH2: dosador de disco alveolado 2; DMS: diferença mínima significativa; médias seguidas de mesma letra minúscula na coluna não diferem pelo teste de Tukey a 5\% de probabilidade de erro; ns: não significativo.

do disco. Os dosadores de disco alveolado são mais sensíveis à elevação da velocidade periférica, fato denotado na maior taxa de redução no percentual de espaçamentos aceitáveis com aumento da velocidade.

\section{REFERÊNCIAS}

BOZDOĠAN, A.M. Seeding uniformity for vacuum precision seeders. Scientia Agrícola, v.65, n.3, p.318-322, 2008. Disponível em: <http://www.scielo.br/scielo.php?pid=S010390162008000300013\&script=sci_arttext $>$. Acesso em: 27 nov. 2012.

CELIK, A. et al. Effects of various planters on emergence seed distribution uniformity of sunflower and seed distribution uniformity of sunflower. Applied Engineering in Agriculture, v.23, n.1, p.57-61, 2007. Disponível em: <http://naldc.nal.usda. gov/download/19264/PDF>. Acesso em: 27 nov. 2012.

DIAS, V. de O. et al. Distribuição de sementes de milho e soja em função da velocidade e densidade de semeadura. Ciência Rural, v.39, n.6, p.1721-1728, 2009. Disponível em: <http:// www.scielo.br/pdf/cr/v39n6/a218cr1046.pdf >. Acesso em: 25 nov. 2012.

\section{INTERNACIONAL ORGANIZATION FOR} ESTANDARDIZATION. ISO 7256/1: Sowing equipment -
Methods of test - Part 1: Single seed drills (precision drills). Genève, 1984. 16p.

IVANČAN, S. et al. Effect of precision on the intra-row seed distribution for parsley drill operating speed. Biosystems Engineering, v.89, n.3, p.373-376, 2004. Disponível em: <http:// dx.doi.org/10.1016/j.biosystemseng.2004.07.007>. Acesso em: 27 nov. 2012.

JASPER, R. et al.Velocidade de semeadura da soja. Engenharia Agrícola, v.31, n.1, p.102-110, 2011.Disponível em: <http://www. scielo.br/pdf/eagri/v31n1/v31n1a10.pdf >. Acesso em: 27 nov. 2012.

KARAYEL, D. et al. Mathematical modelling of vacuum pressure on a precision seeder. Biosystems Engineering, v.87, n.4, p.437444, 2004.

KARAYEL, D.; ÖZMERZI, A. Comparison of vertical and lateral seed distribution of furrow openers using a new criterion. Soil and Tillage Research, v.95, p.69-75, 2007. Disponível em: <http:// dx.doi.org/10.1016/j.still.2006.11.001>. Acesso em: 27 nov. 2012.

KLEIN, V.A. et al. Efeito da velocidade na semeadura direta de soja. Engenharia Agrícola, v.22, n.1, p.75-82, 2002.

LIU. W. et al. Impact of planter type, plantingspeed, and tillage on stand uniformity and yield of corn. Agronomy Journal, v.96, n.6, p.1668-1672, 2004. 
PINHEIRO NETO, R. et al.Desempenho de mecanismos dosadores de semente em diferentes velocidades e condições de cobertura do solo. Acta Scientiarum. Agronomy, v.30, n.5, p.611-617, 2008.

REIS, E.F. dos et al. Características operacionais de uma semeadora-adubadora de plantio direto na cultura da soja (Glycine $\max (\mathrm{L}$.) Merril). Revista Ciências Técnicas Agropecuárias, v.16, n.3, p.70-75, 2007.
SANTOS, S.R. dos et al. Espaço livre entre orifícios de discos dosadores e sementes de milho na eficiência de semeadura. Engenharia Agrícola, v.24, n.1, p.150-157, 2003.

YAZGI, A.; DEGIRMENCIOGLU, A. Optimization of the seed spacing uniformity performance of a vacuum-type precision seeder using response surface methodology. Biosystems Engineering, v.97, p.347-356, 2007. Disponível em: <http://dx.doi.org/10.1016/j. biosystemseng.2007.03.013>. Acesso em: 27 nov. 2012. 